\title{
Outcomes of icotinib combined with concurrent chemoradiotherapy in locally advanced cervical cancer
}

\author{
Yan-Mei LIU ${ }^{1, *}$, Hui LIU ${ }^{2, *}$, Hua-Li SUN ${ }^{1}$, Yuan-Yuan XU11, Yan DING ${ }^{1}$, Wei-Jun CHEN ${ }^{1}$, Shen-Peng YING ${ }^{1, *}$ \\ ${ }^{1}$ Department of Radiation Oncology, Taizhou Central Hospital, Taizhou, Zhejiang, China; ${ }^{2}$ Department of Radiation Oncology, The First Peo- \\ ple's Hospital of Yancheng; The Fourth Affiliated Hospital of Nantong University, Yancheng, Jiangsu, China \\ ${ }^{*}$ Correspondence: yingsp@tzzxyy.com \\ ${ }^{*}$ Contributed equally to this work.
}

Received November 22, 2020 / Accepted February 2, 2021

\begin{abstract}
Concurrent chemoradiotherapy (CRT) based on cisplatin is recognized as the current standard treatment for locally advanced cervical cancer. The treatment of cervical cancer has reached a plateau in the last 20 years. Previous studies have proven that the epidermal growth factor receptor is correlated with chemo- and radioresistance and treatment failure. Hence, the purpose of this study was to investigate the efficacy and safety of icotinib combined with CRT in the treatment of locally advanced cervical cancer. Eligibility criteria included patients treated in the radiotherapy department of Taizhou Central Hospital of Zhejiang Province for stage IIB to IIIB cervical cancers who had not received anti-tumor treatment before and a performance status of 0 to 2. Patients were given icotinib $125 \mathrm{mg}$ three times a day for 6 weeks, which was one week before the start of radiotherapy (500 centigrays in 28 fractions) and chemotherapy $\left(40 \mathrm{mg} / \mathrm{m}^{2}\right.$ administered weekly for 3-5 cycles). There were 29 patients who completed the I+CRT treatment, and it was tolerated well. The median followup time was 50 months and 27 patients (93.10\%) achieved complete responses. The 5-year cumulative overall survival rate and disease-free survival rate were $58.4 \%$ and $60.9 \%$, respectively. The treatment with I+CRT is safe and effective for locally advanced cervical cancer. As far as we know, this is the first study to report the 5-year survival rate of locally advanced cervical cancer with targeted therapy combined with chemoradiotherapy.
\end{abstract}

Key words: cervical cancer, chemoradiation, icotinib, OS, DFS

Cervical cancer is one of the most common malignant tumors among women in the world. As the third most common cancer among females, it is the fourth leading cause of cancer-related death among women [1]. Despite advances in medical technology and the fact that many cervical cancers can be cured in the early stages by surgery, the prognosis of patients with locally advanced cervical cancer treated by concurrent chemoradiotherapy is still poor [2, 3]. So far, concurrent chemoradiotherapy is still the standard treatment for locally advanced cervical cancer. Although chemoradiotherapy has improved the 5 -year survival rate by $6 \%$, nearly one-third of patients still develop a local recurrence or distant metastasis. Therefore, it is important to develop new treatments to improve the cure rate of cervical cancer.

At the present, studies have shown infection with HPV (human papillomavirus) plays an important role in $99.7 \%$ of cervical cancers [4]. High-risk HPV infection is the most predominant cause of cervical cancer. Although vaccines to prevent infection with HPV are available, they have no thera- peutic efficacy, and the number of HPV-related tumors is not expected to decline significantly until 2040 [5]. Therefore, it is still critical to develop novel therapeutic approaches to treat cervical cancer. It has been proved that HPV can stimulate cell proliferation by binding its early protein E5 to a variety of cell growth factor receptors including epidermal growth factor receptor. HPV can stimulate cell proliferation by binding its early protein E5 to a variety of cell growth factor receptors including epidermal growth factor receptor (EGFR) [6].

EGFR is the expression product of the proto-oncogene c-erbB1, one of the major family members of the receptor HER [6]. In normal cells, the EGFR signaling pathways play a very important role in maintaining function. However, its improper activation or overexpression may also cause or promote the occurrence and development of tumors [7]. Therefore, EGFR has become a target for therapeutic drug research and a target in a variety of malignancies [8, 9]. Erlotinib combined with cisplatin-based concurrent chemo- 
radiotherapy has been tested previously in patients who suffered from locally advanced cervical cancer and tolerable toxicity and significant antitumor activity were reported [10]. Icotinib is a specific epidermal growth factor receptor tyrosine kinase inhibitor (TKI). It is the first small molecule anticancer drug with independent intellectual property rights in China. In its chemical structure, molecular mechanism and efficacy, it is similar to erlotinib, but it has better safety [11]. It has been widely used in advanced non-smallcell lung cancer with a certain effect [12].

In summary, according to the actual situation in China, we developed a regimen comprised of icotinib combined with cisplatin and pelvic radiotherapy (CRT) and evaluated its safety and antitumor activity. According to the previous studies, we referred to the application of icotinib in lung cancer where the recommended dosage of icotinib is $125 \mathrm{mg}$, three times a day.

\section{Patients and methods}

Patients. All cervical cancer patients were selected from the radiotherapy department of Taizhou Central Hospital from January 2010 to December 2015. Patients of the International Federation of Gynecology and Obstetrics (FIGO) stage IIB to IIIB, age from 31 to 73 years, a bidimensional

Table 1. Baseline patient characteristics.

\begin{tabular}{lc}
\hline Characteristic & No. of patients (\%) \\
\hline Age (y) & 56 \\
Median & $31-72$ \\
Range & \\
ECOG & 72.41 \\
$\quad 1$ & 27.59 \\
2 & \\
Cycle of chemotherapy & 27.58 \\
3 & 24.14 \\
4 & 48.28 \\
5 & \\
FIGO stage & 58.62 \\
IIB & 3.44 \\
IIIA & 37.93 \\
IIIB & \\
Pathology & 10.34 \\
adenocarcinoma & 89.66 \\
squamous & \\
Tumor grade & 20.69 \\
1 & 48.28 \\
2 & 31.03 \\
3 & \\
Lymph node metastasis & 34.48 \\
yes & 65.52 \\
no &
\end{tabular}

Abbreviations: ECOG PS - Eastern Cooperative Oncology Group performance measurable lesion, and an Eastern Cooperative Oncology Group performance status (ECOG) of 0 to 2 were considered eligible. Serum negativity for b-human chorionic gonadotropin was essential for patients of reproductive age. All patients were confirmed pathologically and had not received radiotherapy or chemotherapy before. After a detailed understanding of the procedure and the advantages and disadvantages of the experiment, all the enrolled patients signed informed consent before the treatment began. This study was approved by the Ethics Committee of Taizhou Central Hospital before it was started.

Exclusion criteria: leukocyte $<3,500$ cells $/ \mathrm{mm}^{3}$ or neutrophil count $<1,500$ cells $/ \mathrm{mm}^{3}$, hemoglobin $<8 \mathrm{mg} / \mathrm{l}$, platelet count $<100,000 / \mathrm{mm}^{3}$, creatinine $>150 \mu \mathrm{mol} / \mathrm{l}$, or estimated creatinine clearance $<60 \mathrm{ml} / \mathrm{min}$, alanine aminotransferase $($ AST) $>75 \mathrm{U} / \mathrm{l}$, aspartic acid amino transferase $($ ALT) $>60 \mathrm{U} / \mathrm{l}$, human albumin $<30 \mathrm{~g} / \mathrm{l}$. In addition, patients diagnosed with another malignancy in the past five years, and those with mental illness, heart disease or human immunodeficiency disease, which are suitable for concurrent chemoradiotherapy, were also excluded.

Endpoints and assessments. The main endpoint of the study was the objective response rate, which refers to the proportion of patients with tumor shrinkage reaching a certain amount and maintaining a certain time, including cases with complete response (CR) and partial response (PR). The secondary endpoint was disease-free survival (DFS), which was defined as the time from the beginning of treatment to the first recurrence or metastasis of the tumor or death of the subject for any reason, and the overall survival rate (OS) was the time from the beginning of treatment to the death due to any cause, and the safety and tolerance of the combined treatment.

All patients need to provide complete medical history before joining the group, and those who meet the requirements need to undergo a comprehensive physical examination, including blood routine, liver and kidney function, myocardial enzyme spectrum, chest and abdomen CT, electrocardiogram, pelvic magnetic resonance imaging and bone ECT, etc.

In addition, during treatment, a gynecological examination and laboratory assessments were given once a week to monitor any side effects. Adverse reactions were classified according to the National Cancer Institute general toxicity Standard Version 3.0. Pelvic magnetic resonance imaging (MRI) was performed after 4 times of brachytherapy to determine the efficacy. If the tumor has achieved complete response, one more brachytherapy will be given; otherwise, another three times should be given. The patients were followed up 1 month after the end of treatment, every 3 months for the first 2 years, and every 6 months in the following 3 years. Clinical and gynecological examinations, pelvic magnetic resonance imaging, and abdominal pelvic CT examinations were performed at each visit to evaluate the efficacy and late toxicity. The next follow-up plan was 
determined according to the examination results. At 90 days after completion of the treatment, the response was assessed according to the RECIST and positron emission tomography/CT results.

Icotinib. To achieve a stable blood concentration, patients were given oral icotinib $125 \mathrm{mg}$ three times a day for a total of 6 weeks before the start of concurrent chemoradiotherapy. Icotinib was provided by Zhejiang Beta Pharmaceutical Co. Ltd. (Hangzhou, China).

Radiotherapy. Radiotherapy was conducted in two stages: pelvic treatment at 5040 centigrays (cGy) 28 fractions, 5 days a week, followed by 5 cycles of brachytherapy, twice a week, 600 centigrays prescribed at point A (International Commission on radiation units report 38) [13]. If the pelvic MRI reexamination at the end of treatment indicated that there was still residual tumor, two more brachytherapy treatments were administered. All treatments were completed within 7-8 weeks.

Chemotherapy. Chemotherapy was given concurrently with radiotherapy once a week, comprised of intravenous cisplatin doses of 30-40 mg/m² (maximum dose, $60 \mathrm{mg}$ ) 3-5 times. None of the patients were given adjuvant chemotherapy after treatment.

Statistical analysis. The SPSS 23.0 statistical software was used for statistical analysis. DFS and OS distributions in this study were described by Kaplan-Meier plots and median estimates.

\section{Results}

Patient characteristics. The toxicity and reactivity were evaluated in 29 patients. Their characteristics are shown in Table 1. The median age of the patients was 56 years (range, 31 to 72 years). In all patients, stage IIB accounted for $58.62 \%$, stage IIIA in $3.44 \%$, and stage IIIB in $37.93 \%$. All patients were treated with concurrent cisplatin chemotherapy, among which 8 (27.58\%) patients completed three cycles, 7 (24.14\%) finished four cycles, and 14 (48.28\%) five cycles.

Patient response. The median follow-up time was 50 months (range 10-80 months). The activity of the therapy was analyzed in 29 patients, all of whom achieved an objective response after the combination treatment. Twenty-one patients $(72.41 \%)$ achieved a CR, whereas 8 patients $(27.59 \%)$ achieved a PR, and 4 (13.79\%) patients achieved a CR one month later. Two of the remaining four patients achieved a CR after 2 rounds of brachytherapy.

At the cut-off date of June 30, 2019, the 5-year DFS and OS rates were $58.4 \%$ and $60.9 \%$, respectively. Figure 1 presents the Kaplan-Meier estimates of DFS and OS for the population. The 5-year DFS and OS were both $76.5 \%$ for FIGO II, and $34.1 \%$ and $36.4 \%$ for FIGO III, and there was a statistical difference in DFS between stage II and stage III ( $p=0.049)$, but no statistical difference in OS ( $\mathrm{p}=0.055$ ) (Figures 2A, 2B). The 5 -year DFS and OS were both $77.8 \%$ for patients without lymph node metastasis, and $48.2 \%$ and $52.6 \%$ for lymph node

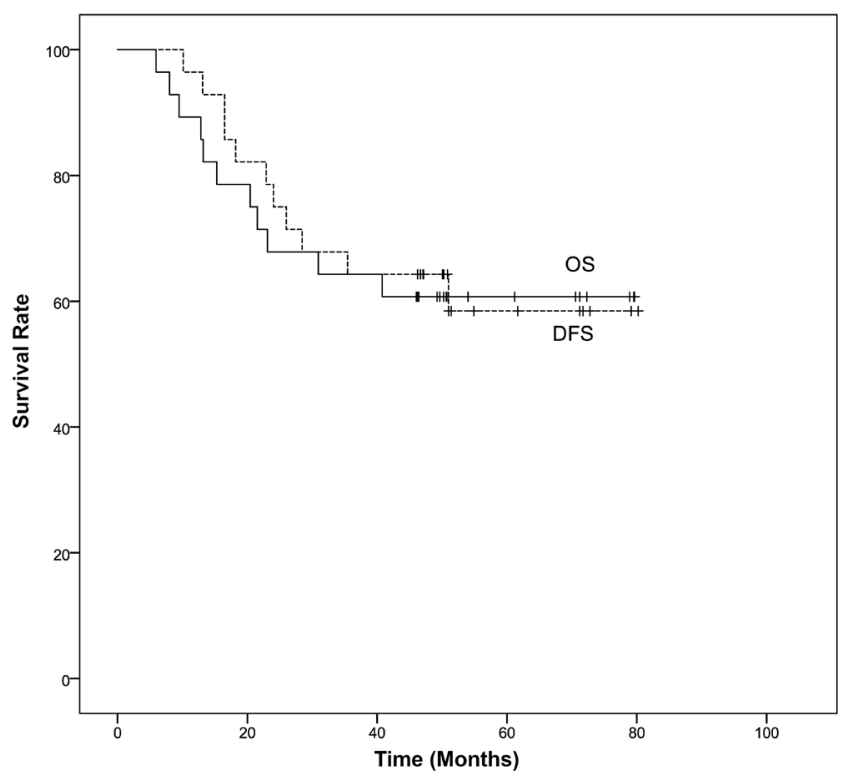

Figure 1. Kaplan-Meier plots for disease-free survival (DFS) and overall survival (OS).

Table 2. Failure patterns of cervical cancer.

\begin{tabular}{lll}
\hline Recurrence pattern & Cases & \% \\
\hline Overall & 11 & 37.93 \\
Cervical & 1 & 3.45 \\
Pelvic lymph node & 2 & 6.90 \\
Paraaortic lymph nodes & 2 & 6.90 \\
Lung & 4 & 13.79 \\
Abdominal organs & 1 & 3.45 \\
Other & 1 & 3.45 \\
\hline
\end{tabular}

metastasis, respectively, the statistical difference was not found in OS $(\mathrm{p}=0.220)$ and DFS ( $\mathrm{p}=0.234)$ (Figures 3A, 3B).

At the end of the follow-up, $11(37.93 \%)$ patients had a disease recurrence or progression, and all of them died. Table 2 shows the number of patients with different types of recurrences. The median recurrence time was 20 months (range 6-40 months). A total of 11 patients recurred, including 5 (17.24\%) cases of pelvic recurrence, 4 (13.79) cases of lung metastasis, 1 (3.45\%) case of duodenal metastasis, and 1 (3.45\%) case of heart disease. Nine (31.03\%) patients had recurrence or metastasis by 2 years.

Toxicity. The toxicity of the 29 patients is shown in Table 3. The most common AEs were diarrhea, nausea, myelosuppression, and radiodermatitis, which were grade 1 or 2 in the majority of patients. Overall, grade 3 toxicities included rash in 1 patient, diarrhea in 8 patients, nausea in 2 patients, radiodermatitis in 1 patient, and myelosuppression in 9 patients. It was found that I-CRT did not aggravate the toxicity, and there were not any therapy-related deaths reported. It is exciting that all patients completed the planned schedule and any side effects of the treatment resolved quickly. 

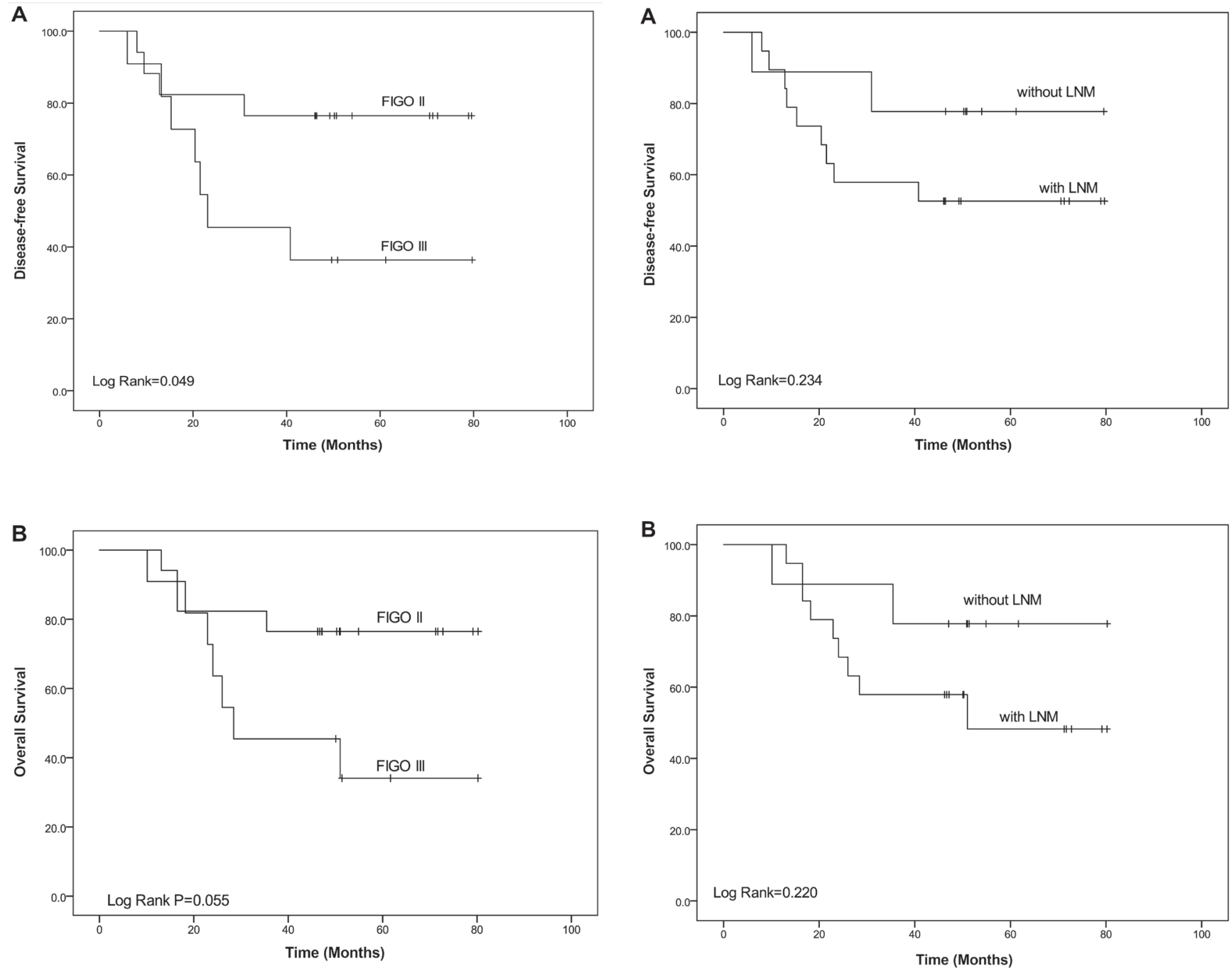

Figure 2. A) Kaplan-Meier plots for disease-free survival of FIGO II and III, B) Kaplan-Meier plots for overall survival of FIGO II and III.

Figure 3. A) Kaplan-Meier plots for disease-free survival of LNM, B) Kaplan-Meier plots for overall survival of LNM.

Table 3. Acute adverse events happened in therapy.

\begin{tabular}{|c|c|c|c|c|c|}
\hline & Grade 0 & Grade 1 & Grade 2 & Grade 3 & Grade 4 \\
\hline Rash & $20(68.97 \%)$ & $3(10.34 \%)$ & $6(20.69 \%)$ & $1(3.4 \%)$ & 0 \\
\hline Diarrhea & 0 & $1(3.4 \%)$ & $20(68.97 \%)$ & $8(27.59 \%)$ & 0 \\
\hline Abnormal liver function & $14(48.28 \%)$ & $12(41.38 \%)$ & $3(10.34 \%)$ & 0 & 0 \\
\hline Nausea & $1(3.4 \%)$ & $22(75.86 \%)$ & $5(17.24 \%)$ & $2(6.90 \%)$ & 0 \\
\hline Myelosuppression & 0 & 0 & $19(65.52 \%)$ & $9(31.03 \%)$ & $1(3.4 \%)$ \\
\hline Radiodermatitis & 0 & $15(51.72 \%)$ & $13(44.83 \%)$ & $1(3.4 \%)$ & 0 \\
\hline
\end{tabular}

\section{Discussion}

Cervical cancer is a common gynecological tumor, being the second most common malignant tumor of the female reproductive system. There are more than 500,000 new cases every year in the world while developing countries account for approximately $85 \%$ [14]. In China, the incidence of cervical cancer is still very high. Nearly 130,000 cervical cancer patients are diagnosed every year, which accounts for one-third of the world's total, and 60,000 people die of cervical cancer [15]. As in other malignant tumors, the development of cervical cancer is a complex multistep process involving multigene regulation. At present, the incidence of cervical cancer has decreased and mortality has been improved with 
the improvement of sanitary conditions and the extensive screening for cervical cancer. However, nearly one-third of patients will have a local recurrence or distant metastasis, and especially in developing countries, cervical cancer is still a common cause of death among women [16]. Therefore, the current work has focused on further improvement of the cure rate and survival of cervical cancer and to find the best treatment plan for cervical cancer.

At present, it is recognized that the main cause of cervical cancer is human papillomavirus (HPV) infection. Most cervical cancer patients with HPV infection are caused by persistent HPV infection, and the human immune system cannot clear HPV. It has been proved that HPV E6 and E7 protein expression is the key immune regulator of host signal transduction and cell transport. When HPV invades the human body, the body responds to the virus. This process involves a variety of signal pathways, such as phosphatidylinositol-3-kinase/protein kinase B (PI3K/AKT) signal pathway, transforming growth factor- $\beta$ (TGF- $\beta$ ) signal pathway, cell death tumor necrosis factor (cell death TNF) signal pathway, cell death Extracellular matrix receptor interaction (ECM receptor interaction) signal pathway. Although the cervical cancer vaccine has been used in clinical practice, the number of HPV-related tumors is not expected to decline significantly until 2040 [5]. Moreover, there are still some HPV-negative cervical cancer patients who need to explore new treatment directions.

In recent years, molecular targeted therapy has become an important part of cancer treatment. At present, clinical research on epidermal growth factor (EGFR) and vascular endothelial growth factor (VEGF) is in full swing. Previous studies [17] have confirmed EGFR is frequently overexpressed in cervical cancer, and so far, the research data has been extremely inspiring [19]. Horowitz et al. [10] suggested that E+CRT therapy is not only safe but also has a significant therapeutic effect on locally advanced cervical cancer. Icotinib is a specific TKI that is the first small molecule anticancer drug with independent intellectual property rights in China. It is similar to erlotinib in chemical structure, molecular mechanism, and efficacy, but better in terms of safety. In addition, a study [2] suggested that icotinib is a potential sensitizer for radiotherapy and cisplatin-based chemotherapy for cervical cancer, which provided the experimental basis for our research.

Based on previous studies and our actual situation, we designed this study. We reported the outcomes of I+CRT in the treatment of locally advanced cervical cancer. Their combination presented a tolerable toxicity profile and favorable outcomes.

We were pleased to find that 21 of $29(72.41 \%)$ patients in our study achieved a CR, and $6(13.79 \%)$ patients achieved a CR two months later, which is $27 / 29$ (93.10\%) achieving a CR. This result is similar to a previous report [10]. After a median follow-up of 60 months, the 5-year DFS and OS rates were $58.4 \%$ and $60.9 \%$, respectively. According to the data presented in the majority of trials, there are four studies reporting the 5-year OS, three of which were similar to ours [19-21]. Wong et al. [22] enrolled 220 cervical cancer patients and $79 \%$ of patients with locally advanced cervical cancer were alive after a median follow-up of 60 months. However, the reason why their study achieved such a high survival rate is that all patients in their study received adjuvant chemotherapy. There is no definite evidence of a clinical benefit for consolidation chemotherapy after concurrent chemoradiotherapy for cervical cancer [23].

According to the previous studies, both staging and lymph involvement are factors affecting the prognosis of cervical cancer [24-26]. As we all know, the FIGO 2018 guidelines include lymph node metastasis in the staging. Matsuo et al. [27] conducted a retrospective study on the SEER research database on data collected in the United States from 1988 to 2014. They found that the prognosis of patients in stage IIIC1 and T3b stage was significantly worse than that of patients without lymph node metastasis. However, the 5-year DFS and OS rates of the current study were $48.2 \%$ and $52.6 \%$, respectively, which seems to be better than theirs. This shows that icotinib combined with concurrent radiotherapy may have a greater survival benefit for patients with lymph node metastasis, although this needs to be confirmed by a further phase III clinical study. Hyo [28] retrospectively analyzed 392 patients and found that the local recurrence rate of advanced cervical cancer (stage III-IV) was significantly higher than that of early cervical cancer $(21.5 \%$ vs. $10.7 \%, \mathrm{p}<0.05)$. The current study found that the 5 -year OS of stage III was $36.4 \%$, significantly lower than that of stage II (75.5\%), which is consistent with previous studies.

The safety of patients is a problem we cannot ignore forever. Diarrhea and myelosuppression are the most common complications of pelvic radiotherapy [29-31], and diarrhea is also one of the common adverse effects of icotinib [32]. In the current study, the incidence of diarrhea and myelosuppression above grade 3 was $27.59 \%$ and $31.03 \%$, respectively, which is similar to the previous results. And all the patients recovered after symptomatic treatment. So, it can be inferred that I+CRT will not cause serious additional adverse effects, their combination is safe and controllable.

As a phase II study, this study has some limitations, including a single institution, a small sample size, no control group, and the possibility of researchers' selection bias. However, it is precisely because it is a single-center study that all of the patients involved were treated by the same team, which avoided the treatment differences caused by the subjective reasoning of medical practitioners. In addition, the follow-up and review of all patients were completed by the same doctor, and the results of that were discussed and decided by the same group, so as to avoid the deviation of follow-up results due to the subjective differences of the follow-up. Finally, because all the patients came from the same area, it was convenient for follow-up. Therefore, none of the patients lost follow-up in our study. 
In conclusion, in the current study of erlotinib combined with cisplatin and radiotherapy as first-line therapy for patients with locally advanced cervical cancer, the treatment combination appears to be safe and shows similar outcomes with previous studies. Additional studies with a larger sample size and randomized controlled studies of this combination are necessary, and they will require a comprehensive evaluation of biomarkers, such as EGFR mutation type. Nevertheless, additional studies need to consider the molecular biological characteristics of cervical cancer to further determine the benefits of combined treatment for patient subgroups and to achieve individualized treatment for this disease.

Acknowledgments: Thanks to my director Mr. Ying for the guidance and design. Thanks to my colleagues for the patient's management and data collection. We are thankful to professor Hu for the study conception and design and to our colleagues for all of their work. The collection, analysis, and interpretation of data of the study were supported by the Taizhou science and technology fund (no.20ywb44).

\section{References}

[1] MILROY MJ. Cancer Statistics: Global and National, pp. 28-35. In: P Hopewood, MJ Milroy. (Eds.) Quality Cancer Care. Springer International Publishing AG, part of Springer Nature 2018. https://doi.org/10.1007/978-3-319-78649-0_3

[2] WANG X, GU Y, LIU H, SHI L, SUN X. Icotinib hydrochloride enhances chemo- and radiosensitivity by inhibiting EGFR signaling and attenuating RAD51 expression and function in Hela S3 cells. Onco Targets Ther 2018; 11: 12451258. https://doi.org/10.2147/OTT.S152613

[3] VACCARELLA S, LORTET-TIEULENT J, PLUMMER M, FRANCESCHI S, BRAY F. Worldwide trends in cervical cancer incidence: impact of screening against changes in disease risk factors. Eur J Cancer 2013; 49: 3262-3273. https:// doi.org/10.1016/j.ejca.2013.04.024

[4] HUGHES C. Cervical cancer: Prevention, diagnosis, treatment and nursing care. Nurs Stand 2009; 23: 48-56; quiz 58. https://doi.org/10.7748/ns2009.03.23.27.48.c6838

[5] Hellner K, Munger K. Human Papillomaviruses as Therapeutic Targets in Human Cancer. J Clin Oncol 2011; 29: 1785-1794. https://doi.org/10.1200/JCO.2010.28.2186

[6] HERBST RS. Review of epidermal growth factor receptor biology. Int J Radiat Oncol Biol Phys 2004; 59: 21-6. https:// doi.org/10.1016/j.ijrobp.2003.11.041

[7] NORMANNO N, BIANCO C, STRIZZI L, MANCINO M, MAIELLO MR et al. The ErbB Receptors and their Ligands in Cancer: An Overview. Curr Drug Targets 2005; 6: 243257. https://doi.org/10.2174/1389450053765879

[8] HOROWITZ NS, OLAWAIYE AB, BORGER DR, GROWDON WB, KRASNER CN et al. Phase II trial of erlotinib in women with squamous cell carcinoma of the vulva. Gynecol Oncol 2012; 127: 141-146. https://doi.org/10.1016/j. ygyno.2012.06.028
[9] BAUMANN M, KRAUSE M, DIKOMEY E, DITTMANN K, DÖRR W et al. EGFR-targeted anticancer drugs in radiotherapy: preclinical evaluation of mechanisms. Radiother Oncol 2007; 83: 238-248. https://doi.org/10.1016/j.radonc.2007.04.006

[10] NOGUEIRA-RODRIGUES A, MORALEZ G, GRAZZIOTIN R, CARMO CC, SMALL IA et al. Phase 2 trial of erlotinib combined with cisplatin and radiotherapy in patients with locally advanced cervical cancer. Cancer 2014; 120: 1187-1193. https://doi.org/10.1002/cncr.28471

[11] SHI Y, ZHANG L, LIU X, ZHOU C, ZHANG L et al. Icotinib versus gefitinib in previously treated advanced nonsmall-cell lung cancer (ICOGEN): a randomised, doubleblind phase 3 non-inferiority trial. Lancet Oncol 2013; 14: 953-961. https://doi.org/10.1016/S1470-2045(13)70355-3

[12] LIU T, JIN L, LU W, GAN H, LIN Z et al. Sequence-dependent synergistic cytotoxicity of icotinib and pemetrexed in human lung cancer cell lines in vitro and in vivo. J Exp Clin Cancer Res 2019; 38: 148. https://doi.org/10.1186/s13046$019-1133-z$

[13] NOGUEIRA-RODRIGUES A, CARMO CC, VIEGAS C, ERLICH F, CAMISÃO C et al. Phase I trial of erlotinib combined with cisplatin and radiotherapy for patients with locally advanced cervical squamous cell cancer. Clin Cancer Res 2008; 14: 6324-6329. https://doi.org/10.1158/1078-0432. CCR-07-5112

[14] SHEN XR, FENG R, CHAI J, CHENG J, WANG DB et al. Modeling age-specific cancer incidences using logistic growth equations: implications for data collection. Asian Pac J Cancer Prev 2014; 15: 9731-9737. https://doi.org/10.7314/ apjcp.2014.15.22.9731

[15] HEN W, ZHENG R, BAADE PD, ZHANG S, ZENG H et al. Cancer statistics in China, 2015. CA Cancer J Clin 2016; 66: 115-132. https://doi.org/10.3322/caac.21338

[16] VERMA A, VERMA S, VASHIST S, ATTRI S, SINGHA A. A study on cervical cancer screening in symptomatic women using Pap smear in a tertiary care hospital in rural area of Himachal Pradesh, India. Middle East Fertility Society Journal 2017; 22: 39-42. https://doi.org/10.1016/j. mefs.2016.09.002

[17] LI Q, TANG Y, CHENG X, JI J, ZHANG J et al. EGFR protein expression and gene amplification in squamous intraepithelial lesions and squamous cell carcinomas of the cervix. Int J Clin Exp Pathol 2014; 7: 733-741.

[18] DAI Q, LING YH, LIA M, ZOU YY, KROOG G et al. Enhanced sensitivity to the HER1/epidermal growth factor receptor tyrosine kinase inhibitor erlotinib hydrochloride in chemotherapy-resistant tumor cell lines. Clin Cancer Res 2005; 11: 1572-1578. https://doi.org/10.1158/1078-0432. CCR-04-0993

[19] Whitney C, Sause W, Bundy BN, Malfetano JH, Hannigan $\mathrm{EV}$ et al. Randomized comparison of fluorouracil plus cisplatin versus hydroxyurea as an adjunct to radiation therapy in stage IIB-IVA carcinoma of the cervix with negative paraaortic lymph nodes: A Gynecologic Oncology Group and Southwest Oncology Group study. J Clin Oncol 1999; 17: 1339-1348. https://doi.org/10.1200/JCO.1999.17.5.1339 
[20] PEARSEY R, BRUNDAGE M, DROUIN P, JEFFREY J, JOHNSTON D et al. Phase III trial comparing radical radiotherapy with and without cisplatin chemotherapy in patients with advanced squamous cell cancer of the cervix. J Clin Oncol 2002; 20: 966-972. https://doi.org/10.1200/ JCO.2002.20.4.966

[21] LORVIDHAYA V, CHITAPANARUX I, SANGRUCHI S, LERTSANGUANSINCHAI P, KONGTHANARAT Y et al. Concurrent mitomycin C, 5-fluorouracil, and radiotherapy in the treatment of locally advanced carcinoma of the cervix: a randomized trial. Int J Radiat Oncol Biol Phys 2003; 55: 1226-1232. https://doi.org/10.1016/s0360-3016(02)04405-x

[22] WONG LC, NGAN HY, CHEUNG AN, CHENG DK, NG TY et al. Chemoradiation and adjuvant chemoradiotherapy in cervical cancer. J Clin Oncol 1999; 17: 2055-2060. https:// doi.org/10.1200/JCO.1999.17.7.2055

[23] TANGJITGAMOL S, KATANYOO K, LAOPAIBOON M, LUMBIGANON P, MANUSIRIVITHAYA $S$ et al. Adjuvant chemotherapy after concurrent chemoradiation for locally advanced cervical cancer. Cochrane Database Syst Rev 2014; 2014: CD010401. https://doi.org/10.1002/14651858. CD010401.pub2

[24] CHO O, CHUN M. Management for locally advanced cervical cancer: New trends and controversial issues. Radiat Oncol J 2018; 36: 254-264. https://doi.org/10.3857/roj.2018.00500

[25] BAE HS, KIM YJ, LIM MC, SANG-SOO SEO, SANGYOON PARK et al. Predictors of Radiation Field Failure After Definitive Chemoradiation in Patients With Locally Advanced Cervical Cancer. Int J Gynecol Cancer 2016; 26: 737-742. https://doi.org/10.1097/IGC.0000000000000662

[26] LIU YM, NI LQ, WANG SS, LV QL, CHEN WJ et al. Outcome and prognostic factors in cervical cancer patients treated with surgery and concurrent chemoradiotherapy: a retrospective study. World J Surg Oncol 2018; 16: 18. https:// doi.org/10.1186/s12957-017-1307-0
[27] MATSUO K, MACHIDA H, MANDELBAUM RS, KONISHI I, MIKAMI M. Validation of the 2018 FIGO cervical cancer staging system. Gynecol Oncol 2019; 152: 87-93. https://doi.org/10.1016/j.ygyno.2018.10.026

[28] BAE HS, KIM YJ, LIM MC, SEO SS, PARK SY et al. Predictors of Radiation Field Failure After Definitive Chemoradiation in Patients With Locally Advanced Cervical Cancer. Int J Gynecol Cancer 2016; 26: 737-742. https://doi.org/10.1097/ IGC.0000000000000662

[29] WANG Y, KONG W, LV N, LI F, CHEN J et al. Incidence of radiation enteritis in cervical cancer patients treated with definitive radiotherapy versus adjuvant radiotherapy. Cancer Res Ther 2018; 14: S120-S124. https://doi.org/10.4103/09731482.163762

[30] PU J, QIN SS, DING JX, ZHANG Y, ZHU WG et al. A randomized controlled study of single-agent cisplatin and radiotherapy versus docetaxel/cisplatin and radiotherapy in highrisk early-stage cervical cancer after radical surgery. J Cancer Res Clin Oncol 2013; 139: 703-708. https://doi.org/10.1007/ s00432-013-1373-9

[31] YUAN Y, YOU J, WANG W, LI X. Long-term follow-up of volumetric modulated arc therapy in definitive radiotherapy for cervical cancer: A single-center retrospective experience. Radiation Medicine and Protection 2020; 2: 81-87. https:// doi.org/10.1016/j.radmp.2020.04.002

[32] XU L, QI Q, ZHANG Y, CUI J, LIU R et al. Combination of icotinib and chemotherapy as first-line treatment for advanced lung adenocarcinoma in patients with sensitive EGFR mutations: A randomized controlled study. Lung Cancer 2019; 133: 23-31. https://doi.org/10.1016/j.lungcan.2019.05.008 